\title{
Generación de contenidos digitales para la reactivación del patrimonio arquitectonico. Estudio de caso: plaza de mercado de techo cubierto de Guayaquil, Medellín*
}

\author{
María Isabel Zapata Cárdenas" - Edwin Mauricio Hincapié Montoya”.. \\ Christian Andrés Díaz León ${ }^{* \cdots *}$ - Camilo Mesías Hoyos ${ }^{* \cdots *}$
}

Recibido: mayo 20 de 2014 - Aceptado: septiembre 13 de 2014

\begin{abstract}
Resumen
Gracias a recursos propios de la tecnología y la convergencia de medios como textos interactivos, datos que permiten navegación táctil, mapas, planos, imágenes y modelos virtuales, entre otros, el patrimonio material puede ser celebrado, contemplado y admirado presionando una tecla. La recreación gráfica de una edificación histórica como fue la Plaza de Mercado de Techo Cubierto de Guayaquil, caso de estudio de esta investigación y recinto de ciudad conocido coloquialmente como la Plaza Cisneros de Medellín, fue un hito social del siglo XIX, pues por su sector se generó todo el movimiento mercantil y del transporte de Medellín. Pero además de esto, fue gracias a la riqueza arquitectónica como se le permitió hacer parte de los bienes de carácter histórico de nuestra ciudad, edificación que años más tarde dejó de existir para dar paso a nuevas obras de desarrollo urbano de la ciudad. Por esto, gracias a la tecnología llamada Realidad Aumentada (RA) y a la creación de contenidos digitales planteados en la investigación, se puede lograr la personalización de la información referente a los patrimonios culturales y su manejo mediante
\end{abstract}

* $\quad$ Este artículo de investigación es publicado como producto de divulgación científica del proyecto "Reactivación del patrimonio arquitectónico del país mediante el uso de la Realidad Aumentada", respaldado por la convocatoria Colciencias para conformar un banco de elegibles "Arte y Cultura". Convocatoria pública N. 570 de 2012. Proyecto de investigación que se encuentra en ejecución hasta el año 2015. Grupo de Investigación Comunicación, Organización y Política COP. Grupo de Investigación Arkadius. Facultad de Comunicación y Facultad de Ingenierías.

** Magister en Periodismo y Comunicación Digital, Universidad Autónoma de Barcelona. Docente tiempo completo Universidad de Medellín. Pertenece al grupo de investigación E-virtual de la Universidad de Medellín. Correo: mizapata@udem.edu.co

*** Doctorado en Ciencias de Ingeniería Model to Reconfiguraion Manufacturing System Based in components. Correo: emhincapie@udem.edu.co

**** Ingeniero biomédico, Escuela de Ingenieria de Antioquia. Doctor of Philosophy (Ph. D.), Modeling, Virtual Environments and Simulation. Correo: christiandiazleon@gmail.com

***** Docente tiempo completo Universidad de Medellín. 


\title{
Generation of Digital Contents to Reactivate Architectural Patrimony. Case Study: “Plaza de Mercado de Techo Cubierto de Guayaquil" in Medellin
}

\begin{abstract}
Thanks to own technological resources and the convergence of media such as interactive texts, data that allow tactile navigation, maps, planes, images, and virtual models, among others, the material patrimony can be celebrated, contemplated, and admired just by pushing a key. Graphic recreation of a historical building, such as "Plaza de Mercado de Techo Cubierto de Guayaquil," study case of this research and city site colloquially known as "Plaza Cisneros de Medellin," was a social milestone of the nineteenth century, since places around this building were the scenarios of marketing and transportations movements in Medellin. For this reason, and thanks to the technology known as Augmented Reality (AR) and the creation of digital contents provided in the research, personalization of information related to cultural patrimony ca be achieved, as well as its handling through mobile devices as mass communication tools by means of which several audiences access contents, navigation routines, and digital information consumption, entertainment experiences, online networking through communication services.
\end{abstract}

Key words: Augmented Reality; architectural patrimony; mobile devices; contents; digital communication.

(viene de la anterior)

dispositivos móviles, como herramientas masivas de comunicación a través de las cuales los diferentes públicos acceden a contenidos, rutinas de navegación y consumo de información digital, experiencias de entretenimiento y relacionamiento en línea a través de los servicios de comunicación.

Palabras clave: Realidad aumentada, patrimonio arquitectónico, móviles, contenidos, comunicación digital. 


\section{Introducción}

Los espacios de significación cultural enriquecen la existencia de los pueblos y proporcionan un profundo sentido, trayendo a escena un pasado que se recupera para la memoria pero que se evidencia y se alimenta por medio de las experiencias compartidas, revelando valores estéticos, históricos, científicos, sociales y espirituales. Perder el patrimonio, olvidarlo e ignorarlo es un gran riesgo para una sociedad que debe construir los estandartes mentales y sociales sobre los que edifica su identidad. El patrimonio cultural es un recurso no renovable en lo que respecta a su pasado, y es por eso mismo que se manifiesta tangiblemente como recurso intocable e irremplazable de un pueblo. De acuerdo con la UNESCO, patrimonio es "el legado que hemos recibido del pasado, lo que vivimos en el presente y lo que transmitimos a las futuras generaciones. Todos los países poseen sitios y monumentos de interés local o nacional pero para que este "patrimonio nacional" sea considerado también "patrimonio mundial" tiene que ser patrimonio de "valor universal excepcional"" (Centro del Patrimonio Mundial, 2014).

Por esto, reactivarlo, traerlo al presente gracias a las herramientas tecnológicas, ponerlo a circular entre los ciudadanos es un trabajo que trasciende en la memoria de "lo nuestro", de la identidad como pueblo y colectividad, y que refuerza los valores como Nación. Pero además de circularlo y traerlo a tiempo presente, el proceso de divulgación y de dar a conocerlo es tan valioso como el patrimonio en sí mismo.

El Ministerio de Cultura de la República de Colombia define al patrimonio como "una categoría legal especial de bienes del patrimonio cultural de la Nación. De este grupo de expresiones, productos y objetos del patrimonio cultural, algunos conjuntos o bienes individuales, debido a sus especiales valores simbólicos, artísticos, estéticos o históricos, requieren un especial tratamiento" (Ministerio de Cultura, 2014).

Esta clasificación fue consignada en la Ley General de Cultura de 1997. Para este caso de estudio, la zona de Guayaquil posee importantes edificaciones que encierran no sólo protagonismo arquitectónico sino social y cultural, que evidencian las diferentes dinámicas, usos económicos y comerciales del Centro de Medellín en el siglo XIX. Por un lado, la vieja Plaza de Mercado estuvo acompañada por los Edificios Carré y Vásquez, que hacen parte de la lista de bienes patrimoniales de Colombia, distinción otorgada por el Ministerio de Cultura del país. La máxima entidad cultural de la Nación, explica que esta distinción se confiere a los

[...] monumentos nacionales y bienes de interés cultural de carácter nacional son el conjunto de inmuebles, áreas de reserva natural, zonas arqueológicas, centros históricos, sectores urbanos y bienes muebles que, por sus valores de autenticidad, originalidad, estéticos, artísticos y técnicos, son representativos para la Nación, constituyéndose además en testimonio vivo de su historia y de su cultura (Ministerio de Cultura, 2014).

En este marco de país, como política pública nacional, que además conversa y se comunica con las políticas municipales y departamentales, el tema patrimonial se extiende a lo local, donde en el caso de Medellín y Antioquia se establece en el Plan de Desarrollo 
de la Alcaldía de Medellín, en su Línea 1: Ciudad que respeta, valora y protege la vida, "Cultura Ciudadana para la Vida", donde se presenta su programa de Memoria y Patrimonio. De acuerdo con el documento publicado Segunda Parte: Diagnóstico-Evaluación y Seguimiento. Plan Especial de Protección Patrimonial de la Alcaldía de Medellín,

[...] el inventario del patrimonio inmueble de la ciudad de Medellín al momento de iniciar el PEPP comprende 137 elementos, de los cuales 30 tienen reconocimiento como bienes culturales del orden nacional. En este conjunto se destaca la presencia dominante de edificaciones individuales; de tipo religioso, institucional y representativo, pertenecientes a distintos momentos de la arquitectura de la ciudad. La arquitectura doméstica tiene una menor participación, y de ella, la gran mayoría se concentra en los barrios Prado, Los Ángeles y el Centro de la ciudad (Alcadía de Medellín, 2006).

Por otro lado, el mismo documento que da pie al estudio y valor de los lugares históricos de Medellín le recomienda al Plan de Ordenamiento Territorial POT tener en cuenta la incorporación de otros bienes inmuebles, 372 bienes adicionales, como parte del legado patrimonial para con los ciudadanos de Medellín. Dentro de este estudio figuran los espacios públicos de interés patrimonial inventariados (área urbana), donde aparece el área de interés de la antigua Plaza de Cisneros, espacio central de investigación del proyecto "Reactivación del patrimonio arquitectónico del país mediante el uso dela Realidad Aumentada".

Este panorama ubica la investigación en un enfoque multidireccional, bajo la mirada de la cultura y la historia, a través de inmuebles patrimoniales que reflejan las dinámicas sociales, económicas y cotidianas de una Medellín en crecimiento. La ciudad escogida es más un pretexto a manera de primera fase para la realización de una aplicación tipo prototipo, que puede ser finalmente replicada y aplicada en cualquier lugar del país.

\section{Guayaquil y la Plaza de Cisneros, expresión de la dinámica social del siglo xix}

El patrimonio es tan importante para una sociedad como vulnerable. De él se sostiene la historia, la identidad y las características de las culturas. Un país que protege su patrimonio es un país democrático, rico e igualitario. Las nuevas generaciones deben tener la posibilidad de disfrutar y aprender sobre lo que han sido y serán en un futuro; y esto se logra gracias a las políticas de cuidado, divulgación y preservación del patrimonio en una Nación. Aquellos lugares que estuvieron y que fueron derruidos pueden traerse hoy a escena gracias a los desarrollos y aplicativos de la tecnología. Para ello la tecnología juega un papel de facilitador mediático a través del cual se puede llegar a diferentes espacios y lugares históricamente importantes pero físicamente inaccesibles por su desaparición.

El proyecto auspiciado por Colciencias y la Universidad de Medellín busca inscribirse geográficamente en un lugar de ciudad importante, porque marcó una época de cambio en los modos de vivir la ciudad, pero que tiene un común denominador: el Centro de Medellín, como eje vertebral de la transformación y cambio en el orden urbano de Medelín. Se propone, entonces, hacer una indagación sobre la vieja Plaza de Mercado o Plaza Cisneros, ubicada en el sector de Guayaquil. 
Cuando se afirma que Guayaquil es hoy un espacio histórico, se quiere decir que sus acontecimientos y sus sucesos, sus espacios, su gente y sus códigos culturales, tienen raíces en el pasado, que éste pasado tiene acción en el presente y que éste último aún puede proyectar una sombra benéfica para el porvenir de sus habitantes (Mejía Londoño, 1991).

Guayaquil fue un sector vital para el comercio y la economía de la Medellín de finales del siglo XIX y comienzos del XX. El mercadeo o comercialización de alimentos ha estado vinculado a la creación de un espacio para ello. Fue en julio de 1892 cuando se hizo una licitación pública para construir una segunda plaza de mercado. Esta es adjudicada al proyecto presentado por Carlos Coroliano Amador (Molina Londoño, 1993). Así fue como se dio el desarrollo urbano y se construyó el monumental edificio, llamado Amador, Cisneros y Guayaquil, más adelante, Mercado de Techo Cubierto de Guayaquil. La empresa de Amador incluyó la venta de los lotes aledaños a la plaza donde hoy están los Edificios Carré y Vásquez, restaurados y rescatados por la Fundación Ferrocarril de Antioquia. A esta zona se le sumaron luego desarrollos como la llegada del primer tren de Berrío en 1914 y la inauguración de la Estación de Cisneros, a la que se unió, a su vez, la estación del tren de Amagá que iba a Buenaventura y Cali. Con todo esto, Guayaquil se convirtió en un puerto donde llegaban los campesinos a comercializar sus productos, así como los turistas, los inversionistas, los mismos pobladores de la ciudad y migrantes, que comenzaron a llegar del campo a las ciudades desde 1920.

La plaza de mercado surge como una necesidad de proveer higiénicamente los alimentos. La promovieron desde sus ideas y acompañaron todo el proceso de adjudicación, construcción y seguimiento posterior, tanto en la plaza de Flórez como en la de Guayaquil. Este es uno de los grandes resultados inmediatos del accionar de los higienistas en la ciudad, pues aportaron el concepto inicial, como el control sobre las condiciones ambientales y arquitectónicas del edificio y las urbanísticas y sanitarias de los alrededores (González Escobar, 2006).

Ese gran cuadrado que estaba conformado por la Plaza de Mercado, la estación del Ferrocarril de Antioquia y los Edificios Carré y Vásquez se tornó en el eje central de la ciudad comercial. Pero fueron dos incendios y la transformación de esta zona, que pasó de su máximo esplendor comercial al ocaso, los que hicieron que la Plaza de Mercado y los edificios históricos terminaran abandonados y olvidados. La Plaza Cisneros sufrió un primer incendio en 1937 y otro en 1978.

El último hecho que marcó el fin de esta era para el sector de Guayaquil y la Plaza Cisneros fue el cierre del Ferrocarril en 1978, traslados continuos de los comerciantes y abarroteros a las diferentes plazas satélite de la ciudad marcaron también el desmembramiento del tejido social, cultural y productivo de Guayaquil (Galindo Muñoz, 2011).

Pero su desaparición fue total cuando la dinámica de la plaza fue remplazada en 1984 por la nueva plaza Minorista José María Villa, que funciona actualmente, mejor conocida como La Minorista. Fueron años de abandono y olvido hasta que en la década del 2000 se gestó un plan de transformación del sector con varios proyectos institucionales dirigidos a la recuperación física, económica y social del lugar. 
La demolición del Pasaje Sucre fue uno de los aspectos que más polémica despertó en torno al proyecto de la Plaza Cisneros, declarado Patrimonio Histórico y Artístico de la Nación, fue defendido como objeto de conservación y restauración por parte de las entidades históricas y culturales competentes (Centro Filial del Consejo de Monumentos Nacionales de Antioquia, Dirección de Patrimonio del Ministerio de Cultura) ante lo cual la administración de ese momento hizo caso omiso: mediante un Decreto 1326 del 11 de diciembre de 2002, la Alcaldía ordenó excluir al Pasaje Sucre del inventario de bienes de interés cultural y autorizó su demolición para construir allí la Biblioteca Temática de Empresas Públicas de Medellín y la Universidad Virtual. Esta construcción será integrada a la Plaza Cisneros (Galindo Muñoz, 2011).

Hoy los terrenos de la antigua plaza de mercado están rodeados por una biblioteca pública (Biblioteca EPM), los edificios Carré y Vásquez -recuperados y restaurados- y la Plaza Cisneros, obra de intervención arquitectónica y artística.

\section{La realidad aumentada y los contenidos patrimoniales}

A mediados de los noventas Miligram et al. (1994) propusieron un concepto llamado el continuo de virtualidad, el cual propone que la realidad y la virtualidad pueden ser combinadas y representadas por medio de un continuo (gráfica 1). En uno de los extremos del continuo se encuentra la realidad, es decir, el espacio donde nos encontramos usualmente. En el otro extremo se encuentra la virtualidad, el cual es un espacio totalmente generado y ficticio, cuyo propósito es hacer creer a los que habitan dicho espacio que es real. El área entre los dos extremos es donde se dan las combinaciones entre virtualidad y realidad, llamado de esta manera realidad mixta. La realidad mixta está compuesta por la RA, donde lo virtual aumenta lo real, y la virtualidad aumentada, donde lo real aumenta lo virtual.

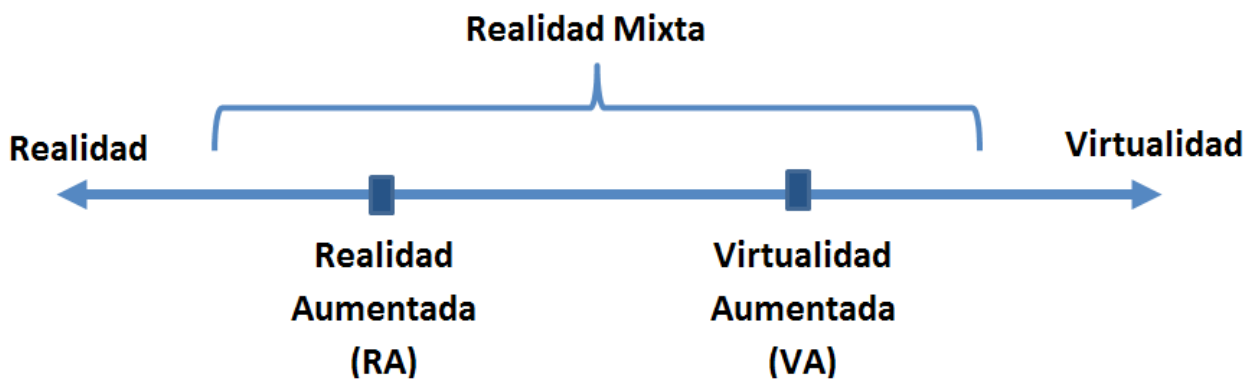

Gráfica 1: Continuo Realidad-Virtualidad propuesto por Milgram 1994

Fuente:

Varios autores han tratado de definir el concepto de realidad aumentada a partir de la definición proporcionada por Miligram et al. (1994). Por ejemplo, Kaufmann (2003) afirma que la RA complementa la realidad en lugar de remplazarla, haciendo que el usuario perciba que los objetos virtuales y la realidad coexisten en un mismo espacio. De manera similar, Azuma 1997 sugiere que la RA debe estar caracterizada por los siguientes factores: 
(i) combinar los objetos virtuales con los objetos reales y (ii) ofrecer al usuario una experiencia interactiva y en tiempo real. En pocas palabras se puede afirmar que la RA busca aumentar la percepción sensorial (visión, audio, tacto, gusto y olfato) que los humanos tienen sobre la realidad. Considerando este último aspecto es donde la RA toma gran importancia como herramienta para la reactivación del patrimonio arquitectónico, ya que permite que las personas puedan percibir sobre el escenario real espacios arquitectónicos y culturales que hoy en día ya no existen porque han sido abandonados o derruidos.

Con este último propósito varios trabajos han propuesto el uso de la RA para la reactivación del patrimonio histórico. Abate et al. (2011) describen el desarrollo de una herramienta que propone el uso de RA para visualizar un guía virtual que asiste a los visitantes en el recorrido por un sitio arqueológico. Kang (2012) propone el desarrollo de una aplicación en RA para dispositivos móviles que reconstruye los sitios históricos y culturales Pitmatgol, Cheonggyecheon y Sewoon localizados en Corea del Sur. Adicionalmente estos autores evalúan cómo distintos paradigmas de interacción afectan el interés y la consciencia que tienen los usuarios sobre la experiencia con los contenidos virtuales. Haugstvedt y Krogstie (2012) proponen el desarrollo de una aplicación de RA para dispositivos móviles que proporciona información histórica, textual y fotográfica, relacionada con las calles de una ciudad. Parte del énfasis de este trabajo está relacionada con la evaluación de la usabilidad de la aplicación y el nivel de aceptación de los usuarios.

Como ha sido mencionado el presente artículo hace uso de la RA para la reactivación del patrimonio arquitectónico de la Plaza Cisneros y sus alrededores. Debido a que los puntos arquitectónicos que se desean reactivar conforman una ruta, se ha decidido utilizar dispositivos móviles como hardware para la visualización del contenido en realidad aumentada. En las gráficas 2 y 3 se puede observar un ejemplo de la aplicación visualizando la locomotora perteneciente al sistema de ferrocarriles (Antioquia) y el tranvía de Medellín.

La aplicación en RA superpone el objeto virtual sobre el escenario real mediante la ejecución de los siguientes pasos:

1. La cámara integrada al dispositivo móvil captura la escena.

2. La aplicación reconoce un patrón o una imagen característica en la escena. Patrón es el nombre que se le da a una imagen predeterminada que se utilizará para determinar en qué punto del escenario real se ubicará el objeto virtual; usualmente esta imagen posee un detalle que contrasta y se diferencia lo suficiente de la escena real.

3. Mediante la implementación de varios cálculos matemáticos la aplicación determina las coordenadas $\mathrm{X}, \mathrm{Y}$ y Z donde está ubicado el patrón y a partir de esa posición dibuja el objeto virtual.

4. La aplicación crea la imagen que combina la escena capturada por la cámara y el objeto virtual superpuesto. Finalmente el dispositivo móvil visualiza la imagen. 


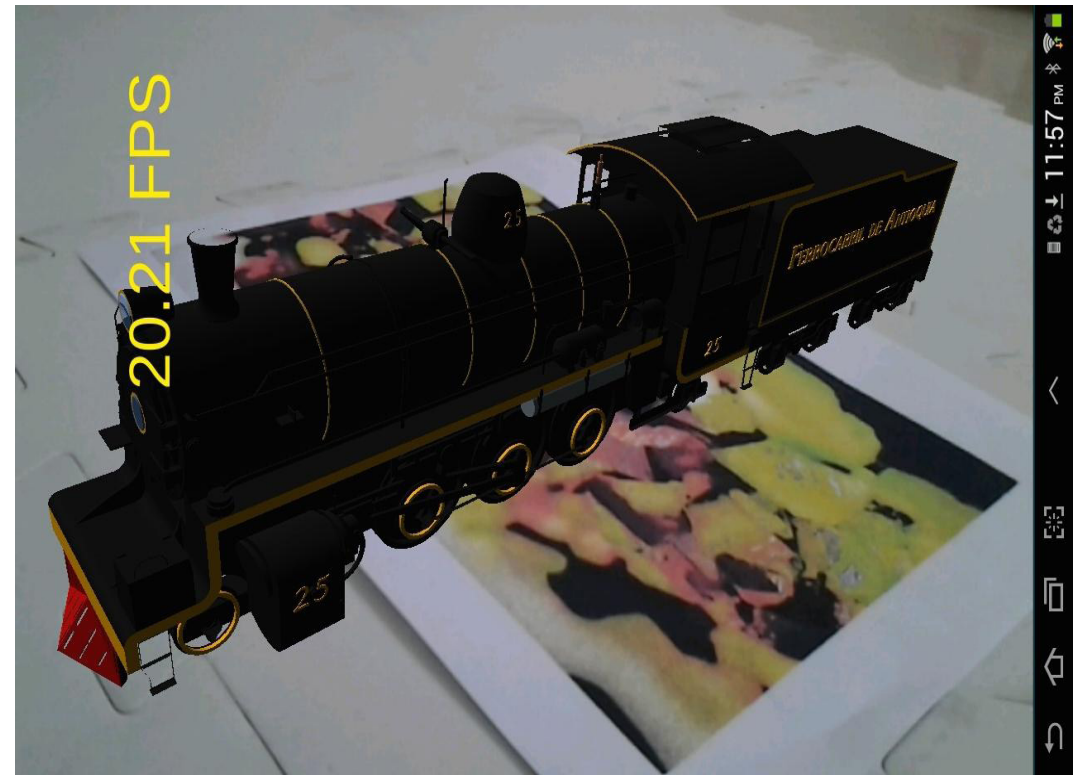

Gráfica 2: Ejemplo del contenido en RA desplegado por la aplicación para la locomotora. Ferrocarril de Antioquia.

Fuente: elaboración propia

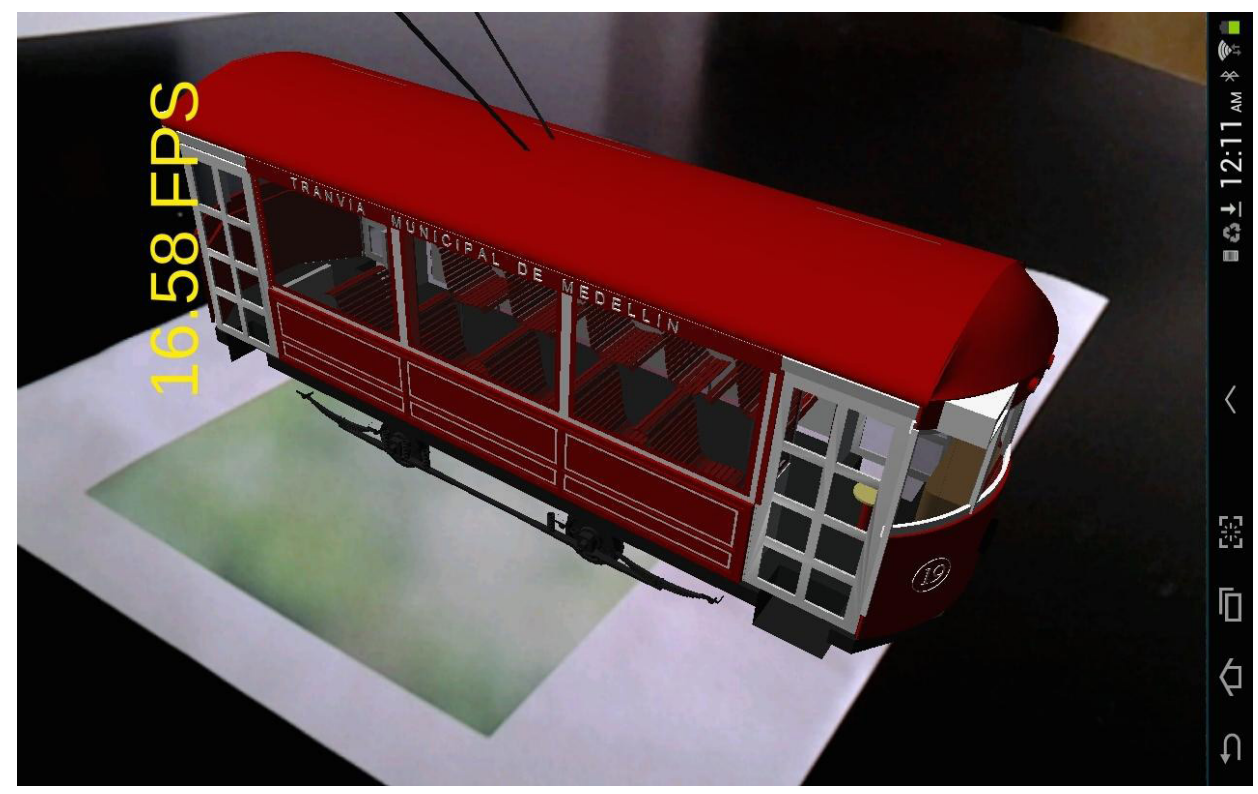

Gráfica 3: Ejemplo del contenido en RA desplegado por la aplicación para el tranvía de Medellín.

Fuente: elaboración propia 


\section{Metodología de la investigación}

Al ser un trabajo con diferentes aristas, la metodología no solo busca generar la experiencia sino desarrollar todo un proceso de construcción, conceptualización, diseño de contenidos tanto comunicacionales como técnicos. Es por esto que la metodología tiene como base los cinco objetivos específicos del proyecto, de manera que pueda darse respuesta a la mixtura de acciones que busca abarcar el proyecto de Realidad Aumentada/Plaza Cisneros. A continuación se explica la metodología desde el enfoque comunicativo y técnico.

\section{Metodología desde el diseño técnico}

Durante la ejecución del proyecto y como resultados más destacables de este se encuentra el desarrollo de una metodología que guía las aplicaciones relacionadas con el patrimonio cultural. Usando 3 elementos: (i) marco de referencia, (ii) matriz de referencia y (iii) guía de aplicación, la metodología determina qué actividades deben desarrollarse, qué herramientas deben utilizarse, cuáles contenidos digitales son apropiados para la aplicación y qué resultados deben obtenerse en cada fase del desarrollo con el fin de maximizar el aprendizaje del patrimonio cultural y la usabilidad de la aplicación.

Esta metodología es el resultado de una revisión del estado del arte, donde se caracterizaron las diferentes aplicaciones desarrolladas en los ámbitos científicos y relacionados con la reactivación del patrimonio cultural, analizando los métodos utilizados para su desarrollo y definiendo las buenas prácticas propuestas por estos trabajos, para construir el contenido de la metodología propuesta. Adicionalmente, se describe cómo ha sido utilizada la metodología mediante su aplicación al caso de estudio de la Plaza Cisneros y sus alrededores. Dentro de los productos y resultados obtenidos están: (i) la recolección de la información histórica que define el contexto en el cual se desarrolla la aplicación, por ejemplo, líneas de tiempo, puntos históricos, eventos importantes, entre otros, (ii) pruebas de campo para valorar el sitio de intervención y de reactivación del patrimonio histórico, (iii) diseño y modelado del dominio del problema y de la aplicación tecnológica para potenciar la reactivación del patrimonio histórico, (v) un prototipo funcional del componente de realidad aumentada que hace parte de la aplicación y que visualiza por el momento la locomotora y el tranvía de Medellín y (vi) una prueba de campo en la cual se evaluaron la visualización del contenido de realidad aumentada en la plaza Cisneros y alrededores, la iluminación, los tipos de marcadores utilizados y la calibración del contenido desplegado con el escenario real, además de registrar los puntos por GPS donde se podrán visualizar los contenidos.

Finalmente, la metodología ha permitido, por medio de las actividades y las herramientas que propone utilizar, dinamizar el desarrollo del caso de estudio, evaluando, de manera anticipada y precisa, aspectos del componente patrimonial y tecnológico.

\section{Metodología desde el diseño de contenidos}

Para la generación de los contenidos digitales, la investigación ha implementado varias etapas o fases del desarrollo de los contenidos de tipo convergentes. Este proceso de 
producción de contenidos digitales demanda pasos de producción complejos, dado que la convergencia incluye múltiples soportes y exige una organización informativa pensada en la interacción y la usabilidad para con el usuario.

Por convergencia de los medios y la creación de contenidos bajo lenguaje digital, Ramón Salaverría expresa que "los nuevos medios reclaman nuevas formas de presentar la información. Las potencialidades hipertextuales e interactivas de las redes digitales exigen a los medios un esfuerzo por desarrollar formatos informativos que aprovechen esas utilidades" (Salaverría, 2003).

Por su parte, García Avilés \& Salaverría (2008) aclaran que la convergencia se desarrolla, al menos, en cinco ámbitos: tecnológico, empresarial, de los contenidos, de los usuarios y de los profesionales de los medios, y que su núcleo no reside ni en las puras transformaciones tecnológicas de la infraestructura de los medios de comunicación, ni en la mera integración de sus equipos y componentes técnicos, sino, sobre todo, en la explotación de los contenidos y servicios, por medio de distintas plataformas de difusión (García Avilés \& Salaverría, 2008).

Para llevar a cabo el diseño, escritura, graficación y producción de los contenidos digitales, se estableció una cadena de producción del contenido digital (tabla 1), mediante la cual se genera una organización de los tiempos, actividades, insumos y productos que cada momento puede requerir.

El desarrollo de los contenidos digitales, técnicos y de la aplicación en RA en sí misma están estrechamente ligados a los objetivos específicos de la investigación que buscan, a grosso modo, la potenciación social del patrimonio. Del grupo de varios objetivos específicos se resalta uno relacionado directamente con el público ciudadano afectado por el proyecto: "proponer un escenario de experiencia entre la tecnología, el patrimonio y la ciudadanía, como aporte al acercamiento a las TIC y la divulgación de la cultura digital".

José María Cuenca López y Myriam Martín Cáceres de la Universidad de Huelva, en su artículo La comunicación del patrimonio desde propuestas de educación no formal e informal, explican que "el patrimonio adquiere un sentido social que justifica plenamente la necesidad de su conservación, al mismo tiempo que deben desarrollarse posturas que apoyen la formación de ciudadanos comprometidos y críticos a partir de esa conformación y respeto identitario" (Cuenca López \& Martín Cáceres, 2014) .

La tecnología actual permite desarrollar propuestas que deben combinar recursos de tipo tradicional con otros que faciliten la interacción y relación directa con el patrimonio; y es en este escenario que la convergencia y los formatos digitales ofrecen posibilidades de interacción, participación, construcción colectiva y gestión de la comunicación y el conocimiento, más aún, cuando el proyecto busca posibilitar al ciudadan-al uso, consulta y apropiación de un hecho histórico por medio de la RA. 
Generación de contenidos digitales para la reactivación del patrimonio arquitectonico. Estudio de caso: plaza ...

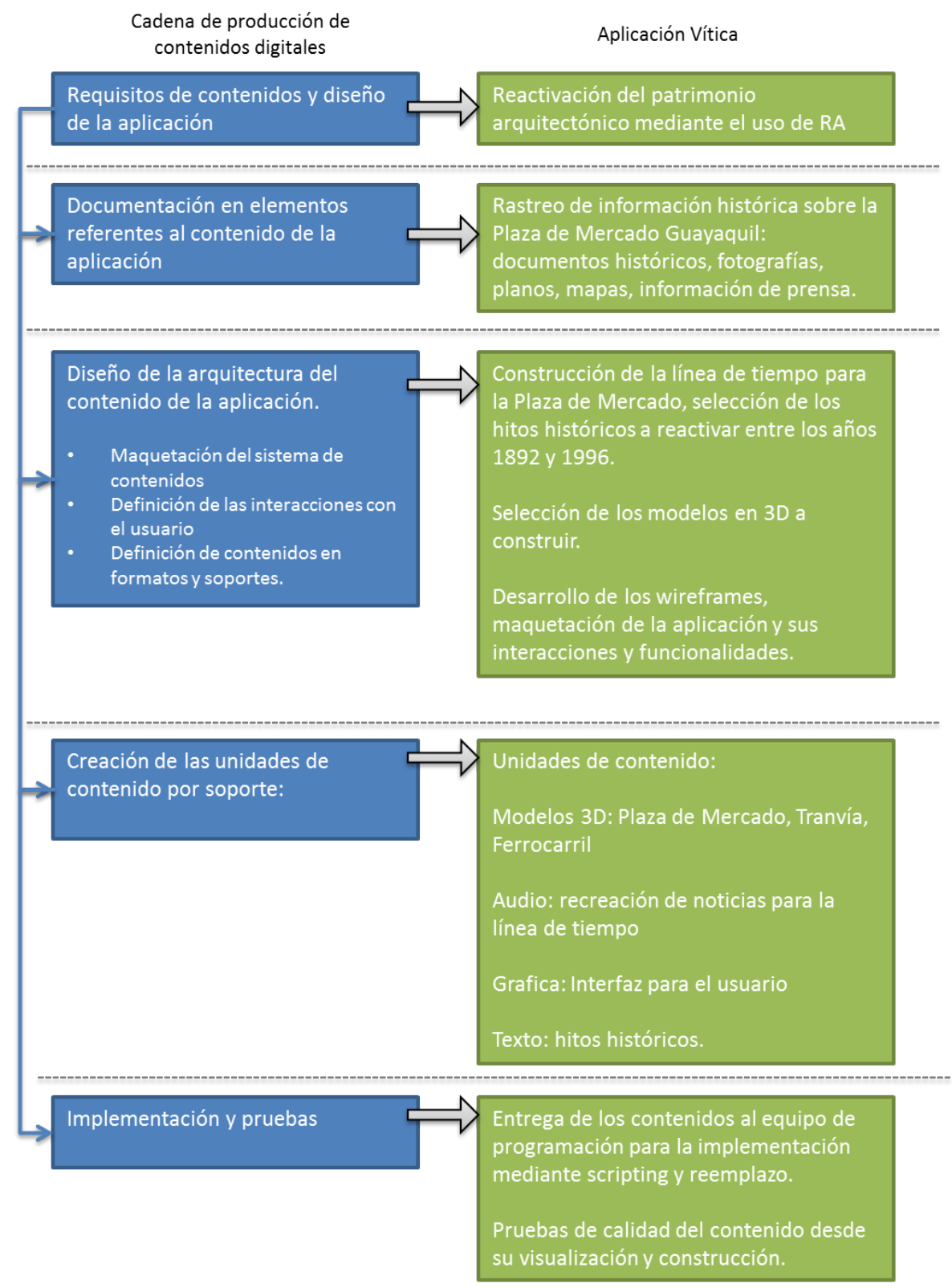

Tabla 1: Etapas generales de la creación de contenidos digitales para Vítica.

Fuente: elaboración propia Igualmente, para una mejor comprensión de la información, los autores Cuenca y Martín reiteran que es importante trabajar los contenidos digitales desde lo actitudinal. Contenidos digitales centrados en el respeto, la interculturalidad y el componente identitario, como referentes básicos de un proyecto de difusión patrimonial. 
Por último, es clave que la estructura de los contenidos le permita al navegante y usuario final conocer hitos clave de los momentos históricos que rodearon la Plaza de Mercado Cisneros, en formatos propios del mundo digital, pero con la fidelidad de las fuentes de información propias del patrimonio, a fin de que el usuario saque sus propias conclusiones y disfrute de un episodio de la historia de su ciudad recreado por las TIC.

En el caso del desarrollo de los contenidos para Vítica, así se denominó el proyecto de Realidad Aumentada para la Plaza Cisneros -Vítica- (Virtualidad, Tecnología y Comunicación); se configuró entonces una cadena de cinco etapas que permitiera el desarrollo del contenido digital.

A continuación se describen las actividades y acciones que cada una de estas etapas requieren:

\section{Primera etapa: requisitos de contenido y diseño}

En esta etapa se analizan los requerimientos que se tienen para los contenidos de la aplicación. Es decir, contrastar los objetivos que se proyectan, los recursos con que se cuenta y el tiempo para establecer cuáles son las limitantes y fronteras de éxito del proyecto. En el proyecto Vítica el principal requisito es la implementación de la RA como mecanismo para la reactivación del patrimonio histórico desaparecido. Esto genera un condicionante inicial: la vinculación de los elementos de RA en el desarrollo del contenido. Como complemento está el carácter histórico del proyecto y, por lo tanto, la necesidad de establecer un contenido que tenga valor y credibilidad desde está mirada.

\section{SEGUNDA ETAPA: DOCUMENTACIÓN}

Durante esta etapa se realizan los procesos de documentación, rastreos bibliográficos e investigación en lo referente a los contenidos. Es la búsqueda de insumos informativos que luego pueden ser empleados como fuentes para la construcción de las unidades informativas. Para la producción de los contenidos de Vítica el rigor histórico de la información es un factor decisivo; por lo tanto, se ha planteado una serie de actividades de consulta de fuentes informativas que incluye las siguientes:

1. Consulta, rastreo y captura de datos en el Archivo Histórico de Medellín

2. Consulta en la Sala de Patrimonio Fotográfico de la Biblioteca Pública Piloto

3. Búsqueda y clasificación de información en la Sala Patrimonial de la Biblioteca de la Universidad de Antioquia

4. Consulta de la Colección de la Fundación Antioqueña de Estudios Sociales (FAES), Centro Cultural Biblioteca Luis Echavarría Villegas. Universidad EAFIT.

Entrevistas focalizadas a historiadores y arquitectos (expertos temáticos) del país. A continuación se relaciona una imagen encontrada en los archivos históricos de la ciudad, con la resolución contractual que dio origen al permiso de construcción de la Plaza de Cisneros en el año 1891. (Ver gráfico 4). 


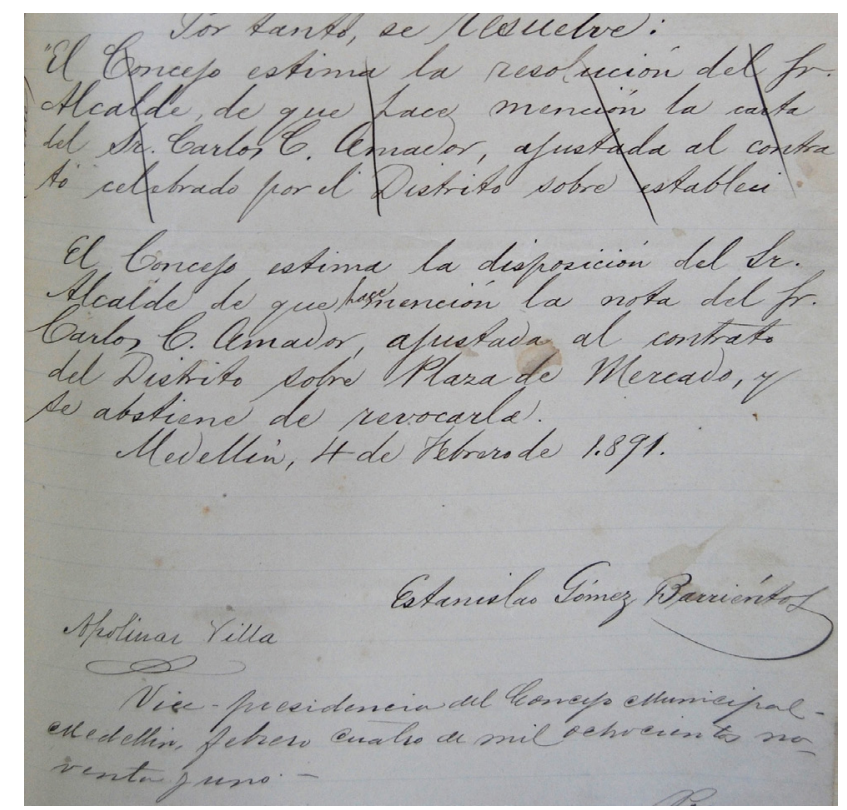

Gráfico 4: Página de las actas oficiales donde se valida la construcción por parte del Alcalde de la Plaza de Mercado Cisneros.

Fuente: Archivo Histórico de Medellín

En esta etapa hay una mezcla de instrumentos para el diseño metodológico y la generación de contenidos como la entrevista focalizada y construcción de fichas bibliográficas donde se consignan los distintos términos, hitos históricos y momentos clave del desarrollo de este lugar, desde su valor social, cultural, comercial y arquitectónico. En esta etapa es clave el diseño de la "ruta", recorrido físico que luego diseña y controla el recorrido por el espacio virtual aplicado a la RA, por donde el usuario podrá descubrir gracias a la información digital aspectos relevantes de la historia e importancia de la Plaza y su incidencia en el ambiente social y mercantil de Guayaquil en el siglo pasado. Para esto se realizó un mapa básico con las calles, distribuciones y edificaciones más importantes de la zona, para así definir qué contenidos serían afectados por la RA, o desarrollados en otros formatos convergentes. (Gráfico 5).

Esta preselección de contenidos y sus formatos fue posible gracias a la clasificación previa en un cuadro que ilustra una línea de tiempo, basándose en los hallazgos y resultados de esta primera y segunda etapa, donde la entrevista con expertos y recorridos por los centros de documentación son claves.

Sobre la entrevista y la muestra de expertos, Sampieri expresa que "en ciertos estudios es necesaria la opinión de individuos expertos en un tema. Estas muestras son frecuentes en estudios cualitativos y exploratorios para generar hipótesis más precisas o la materia prima del diseño de cuestionarios" (Hernandez Sampieri, Fernández Collado, E Baptista Lucio, 2006). 


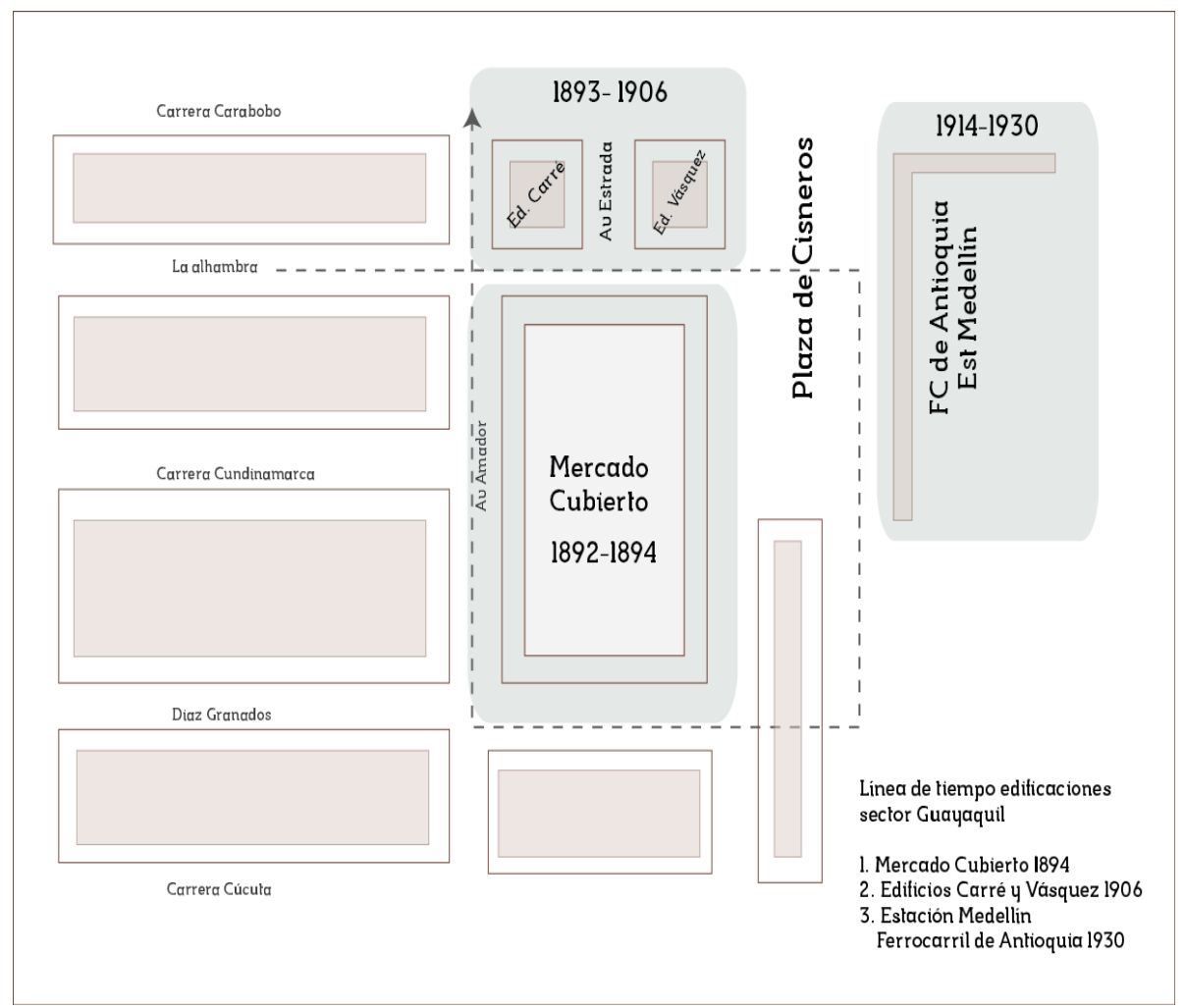

Gráfico 5: Adaptación gráfica plano histórico de Medellín. 1931. Fuente: Centro de Documentación de Planeación (Alpujarra). Medellín.

\section{Diseño: elaboración propia}

La línea de tiempo es la herramienta de graficación de datos óptima, pues gracias a su representación puede adoptar diversas modalidades (lineal, espiral, cíclica, entre otras). La distribución del contenido permite mostrar una relación entre sucesos. En sí, las líneas de tiempo son una manera de contar una historia.

Las líneas de tiempo permiten superponer información e imágenes de forma creativa, configurando un panorama amplio en la representación gráfica de los procesos históricos. Permiten fragmentar en los segmentos que se desea profundizar, destacando aquellos aspectos que considere relevantes (Secretaría de Educación Pública, 2010).

Para el diseño y organización de los contenidos de la línea de tiempo de la Plaza Cisneros se organizó la información de la siguiente manera: (ver tabla 2).

El recurso de la línea de tiempo para la generación de los contenidos digitales es clara y directa, pues permite definir de principio a fin un recorrido por los sucesos más relevantes relacionados con el estudio de caso, la Plaza de Mercado de Techo Cubierto de Guayaquil. 
Tabla 2: Organización de información de algunos momentos importantes relacionados con la Plaza de Mercado Cisneros, para el diseño de los contenidos de la Línea de Tiempo.

\begin{tabular}{|c|c|c|c|c|}
\hline Fecha & $\begin{array}{c}\text { Suceso } \\
\text { texto línea de tiempo }\end{array}$ & $\begin{array}{l}\text { Protagonista } \\
(s)\end{array}$ & $\begin{array}{l}\text { Papel o función del prota- } \\
\text { gonista }\end{array}$ & $\begin{array}{l}\text { Recurso para produc- } \\
\text { ción de contenido }\end{array}$ \\
\hline 1888 & $\begin{array}{l}\text { Documentos legales } \\
\text { para la construcción } \\
\text { de la plaza de mercado } \\
\text { cubierta que reempla- } \\
\text { zaría la existente: Plaza } \\
\text { de Flórez }\end{array}$ & $\begin{array}{l}\text { Carlos } \mathrm{C} . \\
\text { Amador }\end{array}$ & $\begin{array}{l}\text { Propietario de los te- } \\
\text { rrenos en los que se } \\
\text { construye la plaza }\end{array}$ & $\begin{array}{l}\text { Fotografía: Primer } \\
\text { contrato de } 1888 \\
\text { Fuente: Archivo His- } \\
\text { tórico de Medellín }\end{array}$ \\
\hline 1886 & $\begin{array}{l}\text { El tranvía de la ciudad, } \\
\text { cruzando frente a la } \\
\text { entrada principal de la } \\
\text { Plaza. }\end{array}$ & & & $\begin{array}{l}\text { MODELO 3D (Re- } \\
\text { crear Tranvía) } \\
\text { Fotografía histórica } \\
\text { Fuente: Biblioteca } \\
\text { Pública Piloto }\end{array}$ \\
\hline $\begin{array}{l}\text { J un io } \\
27 \text { de } \\
1894\end{array}$ & $\begin{array}{l}\text { Inauguración de la plaza } \\
\text { de mercado cubierta }\end{array}$ & Carlos Carré & $\begin{array}{l}\text { Arquitecto (quien a la } \\
\text { par construía los edi- } \\
\text { ficios Vásquez y Carré, } \\
\text { situados al frente de } \\
\text { la Plaza. Actualmente } \\
\text { restaurados }\end{array}$ & $\begin{array}{l}\text { MODELO 3D (Plaza } \\
\text { de mercado) } \\
\text { Fotografía histórica } \\
\text { Fuente: Biblioteca } \\
\text { Pública Piloto }\end{array}$ \\
\hline 1914 & $\begin{array}{l}\text { Inauguración de la es- } \\
\text { tación Ferrocarril de } \\
\text { Antioquia }\end{array}$ & N/A & N/A & $\begin{array}{l}\text { Modelo 3D de la lo- } \\
\text { comotora del Ferro- } \\
\text { carril de Antioquia } \\
\text { Fotografía histórica } \\
\text { Fuente: Biblioteca } \\
\text { Pública Piloto }\end{array}$ \\
\hline $\begin{array}{l}\text { J u l i o } \\
11 \text { d e } \\
1937\end{array}$ & $\begin{array}{l}\text { Primer incendio en la } \\
\text { Plaza de mercado. Se } \\
\text { ve afectada parte de su } \\
\text { techo y construcción }\end{array}$ & N/A & $\mathrm{N} / \mathrm{A}$ & $\begin{array}{l}\text { Fotografía histórica } \\
\text { Imagen periódico } \\
\text { de la época. Fuente: } \\
\text { Archivo Periódico El } \\
\text { Colombiano }\end{array}$ \\
\hline
\end{tabular}

Fuente: elaboración propia

Para este caso directo, la línea hace énfasis en

1. Establecer la duración necesaria para el contenido: período de tiempo (inicio y fina lización de la línea) de acuerdo a los hechos históricos seleccionados. 
2. Permitir una distribución visual horizontal o vertical de la línea de tiempo. Ideal para las pantallas de celulares.

3. Resaltar las fechas más representativas del suceso, de acuerdo con el trabajo exploratorio por los archivos históricos.

4. Ofrecer un recorrido secuencial, fecha a fecha.

5. Establecer una escritura de textos cortos, claros y concisos.

\section{Tercera etapa: diseño de la arquitectura del contenido}

La principal actividad durante esta etapa es la maquetación del sistema de contenidos, la definición de las interacciones con el usuario y la selección de contenidos según formatos y soportes. Para los requisitos de Vítica es fundamental lograr una clara apropiación del contenido por parte del usuario. Para trasmitir este contenido histórico ha sido necesario implementar elementos que faciliten este contacto entre el usuario y la información a trasmitir. Vincular estrategias como la gamefication puede facilitar ese contacto con el usuario entregándole información en un lenguaje apropiado para el contexto de uso, siendo la transmedia y la convergencia, la naturaleza de un lenguaje apropiado para el espacio digital.

La Narrativa Transmedia son una particular forma narrativa que se expande a través de diferentes sistemas de significación (verbal, icónico, audiovisual, interactivo, etc.) y medios (cine, cómic, televisión, videojuegos, teatro, etc.). La NT no son simplemente una adaptación de un lenguaje a otro: la historia que cuenta el cómic no es la misma que aparece en la pantalla del cine o en la microsuperficie del dispositivo móvil (Scolari, 2013).

Cuando se aborda el tema de los contenidos digitales, no debe mirarse como un tema aislado comprendido solamente del contenido, la estructura gráfica y la información. En la creación de los contenidos digitales se identifican tres factores habilitadores e importantes: la infraestructura, que provee la plataforma física para crear, preservar, distribuir y usar los contenidos digitales; la disponibilidad, entendida como la producción de esos contenidos y su puesta a disposición en el mercado en formato digital; y las habilidades para el acceso y uso de los contenidos digitales, lo que implica un cierto nivel de conocimiento y facilidad para operar los equipos y entender elementos como por ejemplo, las restricciones a la gestión de derechos de propiedad intelectual. Desde este esquema de creación básica de los contenidos digitales, es importante proceder al acercamiento y definición de los contenidos digitales.

De acuerdo con el estudio español "Perfiles profesionales más demandados en el ámbito de los contenidos digitales en España 2012-2017" (Fundación de Tecnologías de la Información (FTI), 2012) se argumenta que la primera aproximación a esa definición debería tener en consideración precisamente el calificativo de digital, por cuanto caracteriza e identifica definitivamente el contenido como tal. Por tanto, la digitalización deriva de la codificación, el empaquetamiento o la simple distribución a través de redes digitales, que necesariamente requieren de la adaptación de unos protocolos específicos. 
Tradicionalmente, los agentes que se dedican a la generación de los contenidos digitales siempre han tenido que ofrecer contenidos a cualquiera que esté interesado, en cualquier momento, en cualquier lugar y en cualquier dispositivo. La consecución última de este objetivo supone la constatación de que el proceso de convergencia tecnológica presenta un impacto real y económicamente relevante para el sector de los contenidos digitales.

De acuerdo con el momento histórico, los contenidos digitales presentan dos notas características: la relevancia económica, esencial para impulsar el desarrollo de la Sociedad de la Información y con ello, contribuir al crecimiento económico del país. Y el potencial de crecimiento, derivado de la digitalización de los contenidos, la multiplicidad de nuevos soportes y la adaptación de las redes de distribución existentes. Acorde con el texto "Tienes 5 segundos", de Juan Carlos Camus, la definición de contenidos digitales, está liderada por autores como "Outing (2002), Paul (2005) y Gillmor (2007) quienes coinciden en destacar que hay aspectos que son exclusivos de los medios de información basados en Internet, tales como la interactividad, la actualización o la permanente incorporación de elementos multimedia. Por eso es que para crear contenidos orientados a esta plataforma, sea necesario realizar esfuerzos diferentes respecto de lo que se hace para otros medios".

Por último, Dan Gillmor (2007), director del Knight Center for Digital Media Entrepreneurship en la Arizona State University, planteó que "los medios son una conversación permanente entre quienes los escriben y sus autores". Por lo mismo recomendaba a quienes estuvieran a cargo de estos, que experimentaran frecuentemente con las nuevas tecnologías, y estuvieron abiertos a las ideas que lleguen; utilizaran las herramientas disponibles; colaboraran con los que participan y tomaran riesgos de manera habitual, ya que "el que fracasa estará mejor preparado la siguiente vez que intente algo'.

En esta tercera etapa, como herramienta para el proceso de maquetación se seleccionó a Cacoo (https://cacoo.com/), una aplicación de dibujo en línea que permite el trabajo colaborativo en el desarrollo de diagramas, tablas de relación, mapas de sitio, wireframes, diseño de interfaces y distribución de contenidos en las pantallas, de acuerdo con las indicaciones técnicas, previamente revisadas por la metodología desde la ingeniería.

En este paso, se realizan los contenidos visuales, como botones de navegación, estructuras visuales de la arquitectura de información, adaptadas a las medidas que por usabilidad y rendimiento se hayan especificado (Ver Gráfico 4).

Además en este proceso, se realiza el levantamiento de un mapa de la zona de interés con el fin de delimitar geográficamente los puntos importantes que deben llevar contenidos digitales, una vez el usuario se encuentre in situ y navegue por la aplicación Vítica. Esto con el fin de determinar qué contenidos y cuáles son los puntos de interés más importantes para realizar los contenidos en diferentes formatos: audios, vídeos, textos, frames con fotografías, ilustraciones 2D y modelos 3D.

1 Camus, Juan Carlos. Gillmor y el cambio de switch. Usando.info. Abril 12, 20014. |ref. de 31 de octubre de 2008] Disponible en Web: http://www.usando.info/blog/2007/10/gillmor-y-el-cambio-de-switch.html 


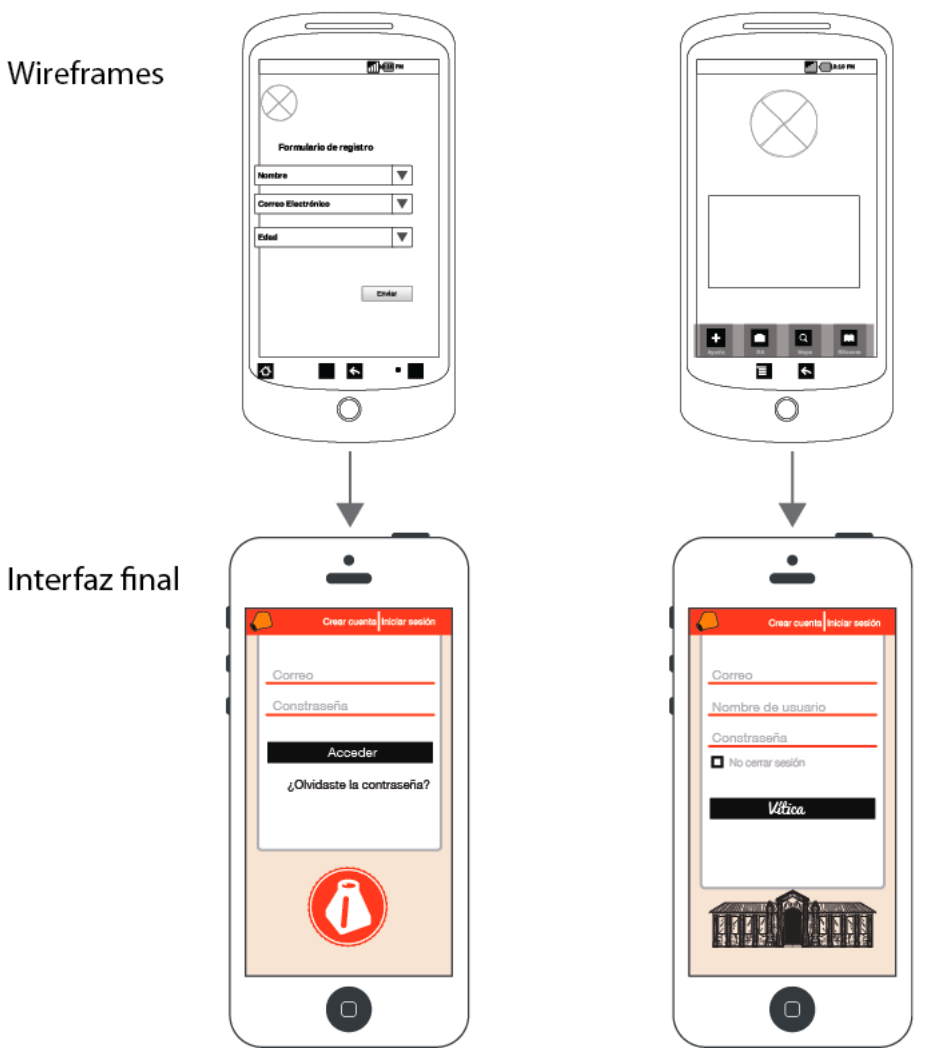

Gráfico 4: Diseño de los componentes visuales de la arquitectura de información.

Fuente: elaboración propia

Para el diseño de este mapa se llevó a cabo un trabajo de campo en el lugar, recorriendo cada uno de los espacios importantes, de la mano de un GPS para que los resultados de geoposicionamiento sean lo más exactos posible, para que el usuario pueda ver con la mayor precisión los contenidos desarrollados. (Ver gráfico 5).

Los puntos marcados con las convenciones RA 1 al 14 son puntos de localización de vista del usuario (desde donde el público navegante podrá ubicarse y detectar los contenidos en sus dispositivos móviles); otros puntos son marcadores y referencias geoespaciales para arrojar los contenidos en 3D.

\section{Cuarta etapa: creación de las unidades de contenido}

Es la etapa en la que se inicia el proceso de construcción propiamente dicho de los contenidos, cada uno en su soporte, tipo de formato, naturaleza gráfica y visual. Se involucra en esta etapa a los realizadores especialistas en cada medio, como pueden ser ilustradores, modeladores, redactores hipertextuales, narradores transmedia, entre otros. En el desarrollo de Vítica la creación de la unidad de contenido de modelado 3D es el 
reto más complejo, pues genera la necesidad de profesionales con alto conocimiento no solo de las técnicas y tecnologías del modelado 3D, sino que deben sumar un alto grado de conocimiento en arquitectura y estilos urbanísticos, lo cual les permite a los modelos tener la pertinencia histórica deseada.

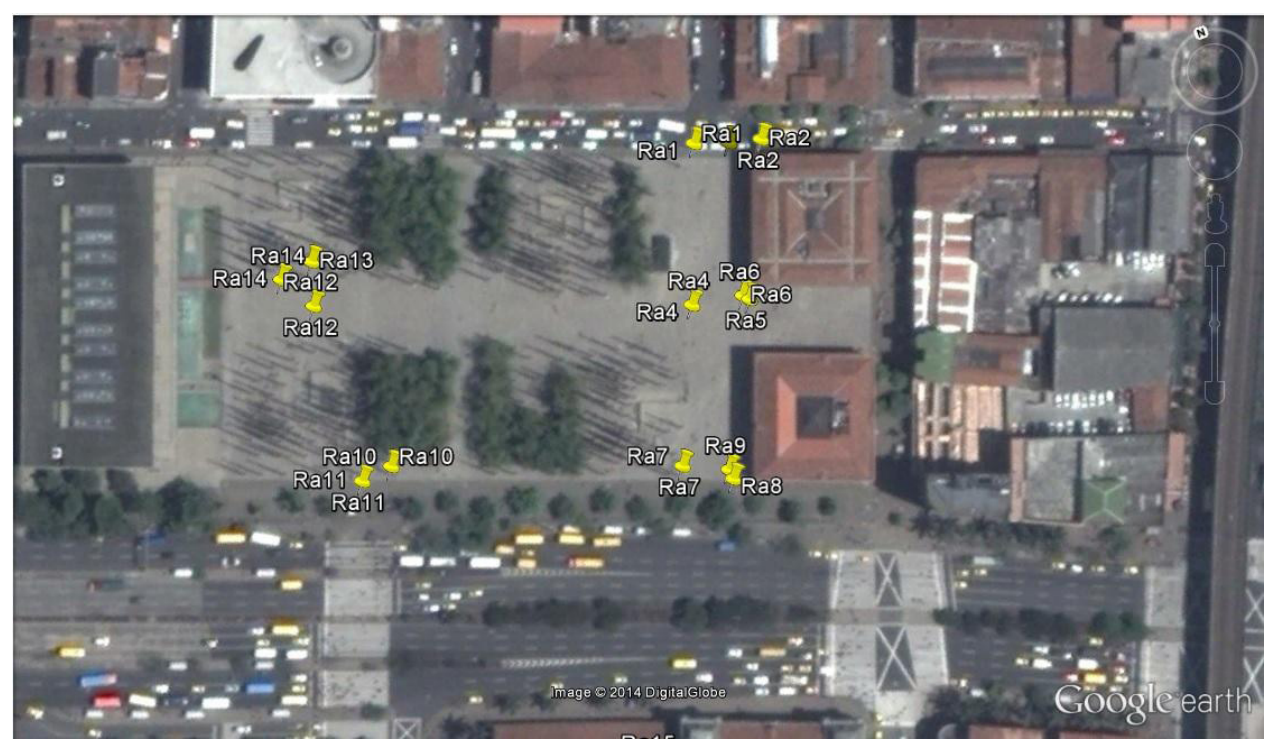

Gráfico 5: Mapa de Google con los puntos referenciados por GPS de la zona de interés, que tendrán contenidos digitales complementarios al desarrollo del modelo Realidad Aumentada.

Fuente: elaboración propia

\section{Quinta etapa: implementación y pruebas}

Una vez cada unidad de contenido ha sido finalizado por su realizador se debe hacer la implementación por parte del equipo programador; buscar la mejor optimización en el uso de la aplicación puede llevar a que muchas veces los contenidos deban ser revisados en elementos técnicos y de configuración, para lo cual el trabajo entre productores de contenidos y programadores debe ser constante y fluido.

Para el desarrollo de la experiencia en RA Vítica la implementación y pruebas de los modelos 3D exigen de un gran trabajo desde la construcción de gráficos de alta calidad y funcionalidad para el usuario, pues los modelos 3D son de difícil comprensión y el peso del archivo generado se puede convertir en una limitante para el funcionamiento de la aplicación en distintos dispositivos móviles.

Autores como Manuel Castells (1997) afirman que las TIC son parte de un proceso en el que se ha venido produciendo una serie de transformaciones apalancadas precisamente por el posicionamientos de estas tecnologías en las dinámicas social, política, económica y cultural. Así, estaríamos en presencia de la emergencia de nuevos escenarios en las 
formas de producción económica, de nuevas formas en la concepción y ejercicio del poder político y también, de la apertura a nuevas formas de sociabilidad debido al uso de las TIC. En este sentido, el desarrollo de las TIC ha sido tan acelerado en los últimos años que las transformaciones en los diferentes ámbitos de nuestro acontecer diario se ven reflejadas en la forma como vivimos y nos relacionamos con nuestro entorno, estableciendo así, nuevas formas de comunicación y de interacción entre los individuos.

Para finalizar, David Ruiz Torres, de la Facultad de Filosofía y Letras de la Universidad de Granada manifiesta que "la Realidad Aumentada también ha demostrado su función pedagógica en otro tipo de escenarios donde constituye uno de los recursos más vanguardistas gracias a que favorece la interacción entre los visitantes y el objeto cultural de una forma atractiva a la vez que didáctica" (Ruiz Torres, 2011).

\section{CONCLUSIONES}

1. Este proyecto de investigación tiene en Medellín y su ruta patrimonial, específicamente el caso de la Plaza de Mercado de Techo Cubierto de Guayaquil un primer acercamiento de prototipo, cuya metodología tanto técnica como de generación de contenidos busca ser aplicada en cualquier item de carácter patrimonial del país.

2. Las técnicas de RA propuestas son formas de representación altamente visuales e interactivas para acceder y comprender los datos histórico-culturales. Estas soluciones tecnológicas no son una alternativa a los entornos reales, sino un valor añadido, de modo que el usuario comprenda sus impresiones y perciba los objetos de manera personalizada sobre diferentes curiosidades y realidades de los recursos comunicacionales y de información de una zona.

3. La metodología ha permitido, por medio de las actividades y las herramientas que propone utilizar, dinamizar el desarrollo del caso de estudio, evaluando de manera anticipada y precisa aspectos del componente patrimonial y tecnológico.

4. El desarrollo de los contenidos digitales para este proyecto está centrado en el respeto, la interculturalidad y el componente identitario, como referentes básicos de un proyecto de difusión patrimonial, y de carácter histórico.

5. El uso de las aplicaciones móviles ha permitido el desarrollo de una experiencia que integra diversos tipos de contenidos digitales como Modelos 3D, audio, video, fotos, texto, entre otros, con propositos de lograr la apropiación de patrimonios históricos y culturales.

\section{Bibliografía}

Alcadía de Medellín. (2006). Alcaldía de Medellín. Obtenido de Alcaldía de Medellín: http://www.google. com.co/url?sa $=$ tErct $=j \xi q=E$ esrc $=$ sEsource $=$ webEcd $=1$ Eved $=0 C B w O F j A A E u r l=h t t p \% 3 A \% 2 F$ \%2Fwww.medellin.gov.co\%2Firj\%2Fgo\%2Fkm\%2Fdocs\%2Fdocuments\%2FServiciosLinea\%2FPlane acionMunicipal\%2FObservatorioPoliticasPublicas\%2FresultadosSeguimiento\%2Fdocs\%2 
Centro del Patrimonio Mundial, U. (01 de Junio de 2014). patrimonio-mundial.com. Obtenido de patrimoniomundial.com: http://www.patrimonio-mundial.com/unescol.htm

Cuenca López, J. M., E Martín Cáceres, M. (19 de Junio de 2014). Academia.edu. Obtenido de Academia. edu: https://www.academia.edu/1493020/La_comunicacion_del_patrimonio_desde_propuestas_ de_educacion_no_formal_e_informal

Fundación de Tecnologías de la Información (FTI). (2012). FTI.es. Recuperado el 3 de Mayo de 2014 , de FTI.es: http://www.fti.es/estudios_informes

Galindo Muñoz, O. (2011). El papel del espacio público en la construcción de la imagen competitiva de la ciudad de Medellín 1998-2007: escalas, imágenes e interacciones. El papel del espacio público en la construcción de la imagen competitiva de la ciudad de Medellín 1998-2007: escalas, imágenes e interacciones. Medellín, Antioquia, Colombia: Universidad Nacional de Colombia, Sede Medellín.

García Avilés, J. A., E Salaverría, R. (2008). La convergencia tecnológica en los medios de comunicación: retos para el periodismo. Trípodos, 31-47.

González Escobar, L. F. (Marzo de 2006). La 'fisiología de la ciudad': médicos, e ingenieros en el Medellín de hace un siglo. Iatreia, 19(1), 77-94.

Hernandez Sampieri, R., Fernández Collado, C., E Baptista Lucio, P. (2006). Metodología de la investigación (Cuarta ed.). México D.F., México: McGRAW HILL IINTERAMERICMA EDITORES, SA DE C.V.

Mejía Londoño, A. (1991). De tacón en la pared. Medellín, Antioquia, Colombia: Ediciones Autores Antioqueños.

Ministerio de Cultura. (01 de Junio de 2014). Icanh. Obtenido de Icanh: http://www.icanh.gov.co/ nuestra_entidad/normatividad/leyes/ley_397_1997_se_dictan_normas_2090

Ministerio de Cultura. (1 1 de Junio de 2014). Sistema Nacional de Información Cultural. Obtenido de Sistema Nacional de Información Cultural: http://www.sinic.gov.co/SINIC/Bienes/PaginaConsultaBienes. aspx?AREID $=3$ ESECID $=10$ ESERID $=$

Ministerio de Tecnologías de la Información y las Comunicaciones. (18 de Marzo de 2014). Colombiatic. Obtenido de Colombiatic: http://colombiatic.mintic.gov.co/602/articles-5550_archivo_pdf.pdf

Molina Londoño, L. F. (3 de Julio de 1993). Banco de la República. Obtenido de Banco de la República: http://www.banrepcultural.org/blaavirtual/revistas/credencial/julio1993/julio3.htm

Ruiz Torres, D. (2011). Realidad Aumentada, educacion y museos. Icono. Revista de Comunicación y Nuevas Tecnologías , 2(14), 212-226.

Salaverría, R. (2003). Convergencia de los medios. Revista Latinoamericana de Comunicación Chasqui, 32-39.

Scolari, C. (2013). Narrativas Transmedia: Cuando todos los medios cuentan. En C. Scolari, Narrativas Transmedia: Cuando todos los medios cuentan (Vol. 1, pág. 341). Barcelona, España: Centro Libros PAPF. Recuperado el 10 de Junio de 2014, de http://www.farq.edu.uy/estetica-diseno-ii/ files/2013/05/1 19756745-1r-Capitulo-Narrativas-Transmedia.pdf

Secretaría de Educación Pública. (2010). Aprende con TIC. Recuperado el 10 de Febrero de 2014, de Aprende con TIC: http://tic.sepdf.gob.mx/micrositio/micrositio3/lineas.html 